\title{
Semaphorin 3A-Plexin-A1 signaling through ERK activation is crucial for Toll-like receptor-induced NO production in BV-2 microglial cells
}

\author{
TAKUJI ITO*, TOKIKO MORITA*, KENJI YOSHIDA, TAKAYUKI NEGISHI and KAZUNORI YUKAWA \\ Department of Physiology, Faculty of Pharmacy, Meijo University, Nagoya 468-8503, Japan
}

Received November 11, 2013; Accepted March 28, 2014

DOI: $10.3892 /$ ijmm.2014.1727

\begin{abstract}
Semaphorin family members have been identified as axonal guidance molecules that mediate the directional determination for axonal elongation during neuronal development. Several semaphorins have been shown to play crucial roles for various immune response phases. In a previous study using knockout mice, we suggested that Plexin-A1, a Semaphorin 3A (Sema3A) receptor, is involved in the increased production of inflammatory factors such as interleukin-1 $\beta$ (IL-1 $\beta$ ) and tumor necrosis factor- $\alpha$ (TNF- $\alpha)$ in the murine microglial response to lipopolysaccharide (LPS). In that study, Sema3A-Plexin-A1 signaling was also shown to have crosstalk with Toll-like receptor 4 (TLR4) signaling to increase nitric oxide production, although the specific intracellular signaling molecule involved in the NO increase was not identified. By investigating the role of Plexin-A1 in the response of the BV-2 microglial cell line to LPS, in the present study novel findings regarding the influence of Plexin-A1 activation on TLR4 signaling in microglial cells were investigated. First, the production of inflammatory markers such as inducible nitric oxide synthase (iNOS), IL-1 $\beta$ and TNF- $\alpha$ in the response to TLR4 stimulation was significantly decreased in BV-2 cells with the knockdown of Plexin-A1. Accordingly, Plexin-A1 was required for the enhanced production of inflammatory factors induced by LPS in BV-2 microglial cells. Second, Plexin-A1 signaling in BV-2 cells showed crosstalk with the LPS-induced TLR4 pathway through activation of nuclear factor- $\kappa \mathrm{B}(\mathrm{NF}-\kappa \mathrm{B})$ and extracellular signal-regulated kinase (ERK). Third, LPS-induced NO production in BV-2 cells was intensified by Sema3A-Plexin-A1 signaling in an ERK1/2 activation-dependent manner. This finding suggested the crucial role of Plexin-A1 signaling through ERK activation in TLR4
\end{abstract}

Correspondence to: Dr Kazunori Yukawa, Department of Physiology, Faculty of Pharmacy, Meijo University, 150 Yagotoyama, Tempaku-ku, Nagoya 468-8503, Japan

E-mail:kyukawa@meijo-u.ac.jp

${ }^{*}$ Contributed equally

Key words: semaphorin, plexin, microglia, lipopolysaccaride activation-induced NO production in BV-2 microglial cells. These results therefore suggest that Plexin-A1 and Sema3A are possible new targets for treating LPS-induced encephalopathy and neuroinflammation-related mental disorders.

\section{Introduction}

Microglia are the resident innate immune cells of the central nervous system (CNS) that regulate neuroinflammation (1). Activated microglia execute host defense and repair injured tissue in the CNS, but they also contribute to the expansion of inflammation in the CNS by means of antigen presentation and release of inflammation-related mediators such as cytokines, chemokines, and nitric oxide (2-4). For example, Toll-like receptor 4 (TLR4), which is expressed in microglia through its ligation with lipopolysaccharide (LPS), has been reported to be involved in microglial activation and cause neuronal injury due to the production and release of inflammation-related mediators (5). However, much remains elusive with regard to how the immunoregulatory molecules controlling microglial function are involved in neuroinflammation.

The roles that semaphorins and their receptors, plexins, play in the CNS have been previously investigated. Semaphorins and plexins comprise two large families of molecules that transmit signals crucial for the control of neuronal axon guidance, cell migration, cell morphology, and cell-to-cell interactions (6). The various functions of semaphorins and plexins have also been exemplified in the development of blood vessels and heart $(7,8)$ and in tumorigenesis $(9)$. Previous investigations have demonstrated a role for these molecules in the immune system (10). For example, Plexin-C1 expressed in dendritic cells moderately affect T-cell activation(11). Plexin-D1, which is highly expressed in double-positive thymocytes, regulates the intrathymic migration of immature thymocytes from the thymic cortex to medulla (12), and Plexin-A4 is a negative regulator in T-cell activation (13). Other studies have revealed the diverse functions of plexins in the immune system. Plexin-A1 expressed in dendritic cells regulates the interaction between dendritic cells and T cells to control adaptive immunity $(14,15)$. Plexin-B1 expressed in microglia and Plexin-A4 in macrophages exhibit a crucial role in promoting the activation of these innate immune cells through the enhancement of intracellular signals initiated by inflammatory stimuli. For 
example, macrophages in Plexin-A4-deficient mice show the significant attenuation of LPS-induced cytokine production (16). Administration of a ligand of Plexin-A4, Semaphorin 3A (Sema3A), to cultured peritoneal macrophages enhances LPS-induced cytokine production in a Plexin-A4-dependent manner (16). Sema4D enhances the CD40-mediated activation of microglia through Plexin-B1 (17).

In a previous study, we showed that Plexin-A1-deficient microglial cells are significantly resistant to LPS-induced inflammation and that Plexin-A1-mediated signaling increased microglial NO production through crosstalk with the LPS-stimulated TLR4 pathway (18). However, the role that Plexin-A1-mediated signaling plays in the expression of other LPS-induced inflammatory mediators in microglia has not been clarified. Similarly, the intracellular signal transduction pathway of Sema3A-Plexin-A1 signaling, which is involved in the increase of LPS-induced microglial NO production remains to be clarified.

Against this background, using a BV-2 microglial cell line, the aim of this study was to examine the intracellular signal transduction pathway of Sema3A-Plexin-A1-mediated signaling, which is involved in LPS-stimulated microglial activation. Additionally, we aimed to demonstrate the necessity of Plexin-A1 for the optimal production of inflammatory mediators following stimulation of the TLR4 pathway in BV-2 cells. In this study, the targeted silencing of Plexin-A1 in BV2 cells resulted in the significantly decreased activation of nuclear factor $-\kappa \mathrm{B}(\mathrm{NF}-\kappa \mathrm{B})$ and of extracellular signal regulated kinase $1 / 2(E R K 1 / 2)$ in the LPS response. Sema3A serves as a ligand for Plexin-A1 in BV-2 cells and enhances LPS-induced NO production in the cell line through ERK activation in a Plexin-A1-dependent manner. Taking these findings together, we propose the functional role of Plexin-A1 as a regulator of the TLR4 pathway in microglia.

\section{Materials and methods}

Cell cultures and transfection. BV-2 murine microglial cells immortalized by infection with v-raf/c-myc recombinant retrovirus (19) were kindly provided by Dr N. Kaneda (Faculty of Pharmacy, Meijo University) with permission to use the cell line from Dr E. Blasi (Department of Diagnostics, Clinical and Public Health Medicine, University of Modena and Reggio Emilia, Modena, Italy). In brief, BV2 cells were maintained in Dulbecco's modified Eagle's medium (DMEM) (Wako, Osaka, Japan) supplemented with $10 \%$ fetal bovine serum (FBS; Equitech Bio, Inc., Kerrville, TX, USA), penicillin (20 U/ml), and streptomycin $(20 \mu \mathrm{g} / \mathrm{ml}$, Sigma-Aldrich, St. Louis, MO, USA). For transfection with small interfering RNA (siRNA), BV-2 cells were seeded in 6-well plates for western blotting, 24-well plates for reverse transcription-polymerase chain reaction (RT-PCR) and quantitative RT-PCR, and 96-well plates for the Griess reaction and 3-(4,5-dimethylthiazol-2-yl)2,5-diphenyl-tetrazolium bromide (MTT) assay at cell densities of $1 \times 10^{5}, 2 \times 10^{4}$, and $0.3 \times 10^{4}$, respectively. Plated cells were grown in DMEM with $10 \%$ FBS overnight, and were then transfected with siRNA-Plexin-A1 (MS0178728; Takara Bio Inc., Shiga, Japan) and siRNA-control (SNC01; Takara Bio Inc.) using the Lipofectamine RNAiMAX transfection reagent (Life Technologies, Carlsbad, CA, USA) $48 \mathrm{~h}$ prior to treatment with reagents such as LPS and Sema3A, according to the manufacturer's protocol.

Mice. Plexin-A1-deficient mice were produced by the gene-targeting method with E14.1 embryonic stem (ES) cells (15). The gene-targeting vector was designed to replace the exon covering the initiation codon with a neomycin-resistance gene and then transfected into ES cells by electroporation. G418 and ganciclovir-resistant clones were screened by PCR and confirmed by Southern blotting. Mutant ES cells were introduced into mouse blastocysts, then transferred into pseudopregnant mice to generate chimeras. Chimeras were bred with $\mathrm{Balb} / \mathrm{c}$ mice to transmit the mutant allele to the germline. Pairs of resultant heterozygous mice were bred to gain homozygous knockout mice, which were backcrossed with Balb/c mice. This study used F10 generation knockout mice, with their wild-type littermates as controls. Mice were housed in the animal facilities of Wakayama Medical University and the animal center of the Faculty of Pharmacy, Meijo University. The care and use of mice and other experimental protocols were conducted in accordance with the guidelines of the Physiological Society of Japan as well as the guidelines on animal experimentation of both Wakayama Medical University and Meijo University. The Animal Ethics Review Committee of both institutions approved the experimental protocol.

Genotype analysis. Genotyping was performed by PCR with mouse tail DNA as the template and a Plexin-A1 gene-specific primer set as previously reported (15).

RT-PCR analysis for Plexin-A1 and Neuropilin-1 gene transcripts. RNA was extracted from primary microglia, the BV-2 microglial cell line, or murine hippocampus by the SV Total RNA Isolation System (Promega, Madison, WI, USA). Reverse transcription of RNA was performed with SuperScript III reverse transcriptase and random primers (Life Technologies). All the samples were normalized with $\beta$-actin amplification for semiquantification. The following oligonucleotides were used for PCR amplification: Plexin-A1, forward: 5'-GTGTGTGGATAGC CATCA-3' and reverse: 5'-CCAGCCTCTCGAACACT-3'; Neuropilin-1, forward: 5'-GGCCTCCTGCGATTCGTTACTGC T-3' and reverse: 5'-CTTAGCCTTGCGCTTGCTGTCATC-3'; and Sema3A,forward: 5'-ATTGAATTCAACTATGCAAACGG AAAGAA-3' and reverse: 5'-TAAGCGGCCGCGACACTTCTG GGTGCCCGCT-3'. Control primers used were: $\beta$-actin, forward: 5'-GGGACGACATGGAGAAGATC-3' and reverse: 5'-AGG TCTTTACGGATGTCAACG-3'. All the primers were annealed at $63^{\circ} \mathrm{C}$, and 35 cycles of amplification were performed.

Western blotting. For western blotting, tissue extracts were prepared by homogenizing murine hippocampal tissue in T-PER Tissue Protein Extraction Reagent (Thermo Scientific Inc., Waltham, MA, USA) containing the protease inhibitor, $\alpha$-complete (Roche Applied Science, Penzberg, Germany). Fifteen micrograms of each sample were adjusted to give a final solution of $60 \mathrm{mM}$ Tris- $\mathrm{HCl}$ (pH 6.8), 2\% SDS, 10\% glycerol, $0.1 \%$ bromophenol blue, and $5 \% \beta$-mercaptoethanol. The solution was heated at $95^{\circ} \mathrm{C}$ for $5 \mathrm{~min}$, electrophoresed through a $10 \%$ SDS-polyacrylamide gel, and transferred to polyvinylidine difluoride membranes (Amersham Pharmacia Biotech, 
Buckinghamshire, UK). Plexin-A1, Neuropilin-1, TLR4, iNOS,

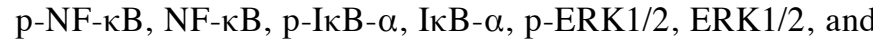
$\beta$-actin were detected with their respective antibodies using an enhanced chemiluminescence western blot detection system (Amersham Pharmacia Biotech) in accordance with the manufacturer's instructions. Antibodies used were anti-Plexin-A1 antibody (Abcam, Cambridge, MA, USA), anti-Neuropilin1 antibody (Abcam), anti-TLR4 antibody (Santa Cruz Biotechnology, Inc., Dallas, TX, USA), anti-iNOS antibody (Merck Millipore, Darmstadt, Germany), anti-p-NF- $\kappa$ B antibody (Cell Signaling Technology, Beverly, MA, USA), anti-NF- $\mathrm{KB}$ antibody (Cell Signaling Technology), anti-p-IкB- $\alpha$ antibody (Cell Signaling Technology), anti-IкB- $\alpha$ antibody (Cell Signaling Technology), anti-p-ERK1/2 antibody (Cell Signaling Technology), antiERK1/2 antibody (Cell Signaling Technology), anti-Sema3A antibody (Abcam), and anti- $\beta$-actin antibody (Cell Signaling Technology).

NO assay and cell viability assay. To investigate the effect of Sema3A on NO production in the BV-2 microglial cell line, the nitrite content was measured with Griess reagent [1\% sulfanilamide/ $0.1 \% \mathrm{~N}$-(1-naphthyl)-ethylenediamine dihydrochloride in $5 \%$ phosphoric acid] according to the manufacturer's instructions. BV-2 microglial cells were plated at $0.3 \times 10^{4}$ cells/well in a 96-well plate and cultured overnight in a $\mathrm{CO}_{2}$ incubator at $37^{\circ} \mathrm{C}$, combined with control siRNA or Plexin-A1-specific siRNA, and incubated for two additional days. Eighteen hours after stimulation of BV-2 cells with LPS and Sema3A or control IgG, $50 \mu \mathrm{l}$ of the culture supernatant were mixed with $50 \mu \mathrm{l}$ of Griess reagent and incubated for $15 \mathrm{~min}$. Absorbance values were measured at $540 \mathrm{~nm}$ in a plate reader (Bio-Rad, Gladesville, NSW, Australia), with fresh DMEM as a blank in all the experiments. NO concentration was calculated with reference to the nitrite standard curve. To analyze cell viability of the BV-2 cells subjected to NO assay, $5 \mu \mathrm{l}$ of MTT $(5 \mathrm{mg} / \mathrm{ml}$; Sigma, Tokyo, Japan) were added to the $\mathrm{BV}-2$ cells and incubated at $37^{\circ} \mathrm{C}$ for $4 \mathrm{~h}$. After the medium was removed, formazan, a product of the MTT tetrazolium ring that was precipitated by the action of mitochondrial dehydrogenases, was dissolved with $0.1 \mathrm{~N} \mathrm{HCl}$ in anhydrous isopropanol containing 10\% Triton X-100 and quantified spectrophotometrically at $595 \mathrm{~nm}$ for measurement of cell viability.

Quantitative RT-PCR for analysis of inflammatory gene transcripts. RNA was isolated from BV-2 microglial cells cultured on a 24-well plate using the SV Total RNA Isolation System (Promega). Quantitative RT-PCR with SYBR-Green (Qiagen, Tokyo, Japan) was performed on a 7500 Fast Real-Time PCR System (Applied Biosystems, Tokyo, Japan). Amplification of Rn18s was performed for sample normalization. For the detection and quantification of Rn18s, TNF- $\alpha$, IL-1 $\beta$, and iNOS transcripts, quantitative RT-PCR was performed using QuantiTect Primer Assays following the manufacturer's protocol (Mm_Rn18s_2_SGQT01036875Mm_Tnf_1_SG QT00104006, Mm_Il1b_2_SG QT01048355, and Mm_iNOS_1_SG QT00100275, respectively; Qiagen).

Reagents for cell cultures. LPS (Escherichia coli serotype 055:B5), Griess reagent, and MTT were obtained from Sigma-Aldrich. Recombinant Sema3A, which consists of the
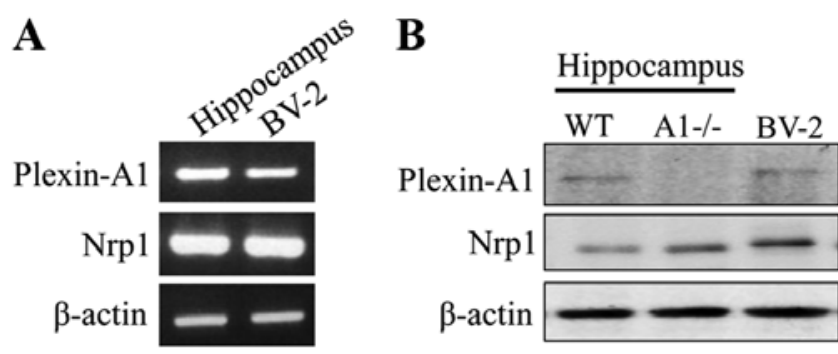

Figure 1. Plexin-A1 and Neuropilin-1 are expressed by BV-2 cells. (A) Reverse transcriptase-polymerase chain reaction (RT-PCR) detects mRNA expression of Plexin-A1 and Neuropilin-1 in murine hippocampus and BV-2 microglial cells. (B) Western blotting detects Plexin-A1 protein in wild-type (WT) but not Plexin-A1-deficient (A1-/-) murine hippocampus. Plexin-A1 protein was also detected in BV-2 cells. Neuropilin-1 was detected in both WT and A1-/- mouse hippocampus and in BV-2 cells. WT, wild-type; A1-/-, Plexin-A1-/-; Nrp1, Neuropilin-1.

extracellular region of Sema3A and the Fc portion of murine $\operatorname{IgG} 2 \mathrm{~A}(\mathrm{Sema} 3 \mathrm{~A}-\mathrm{Fc})$ and murine $\mathrm{IgG} 2 \mathrm{~A}$, was purchased from R\&D Systems (Minneapolis, MN , USA). ERK1/2 inhibitor PD98059 was purchased from Cell Signaling Technology.

Statistical analysis. Data were expressed as the means \pm standard error of mean (SEM). Statistical analyses were performed with the Student's t-test or one-way analysis of variance followed by post-hoc analysis. $\mathrm{P}<0.05$ was considered statistically significant.

\section{Results}

Plexin-Al is expressed by $B V-2$ cells. In a previous study, we found that murine primary microglia expressed Plexin-A1 (18). In the present study, the expression of Plexin-A1 in the BV-2 mouse microglial cell line was confirmed by RT-PCR analysis and western blotting (Fig. 1).

Plexin-A1 is required for $i N O S, I L-1 \beta$ and TNF- $\alpha$ expression in $B V-2$ cells. As in primary microglia, LPS stimulation induces an increased expression of inflammatory mediators such as iNOS, IL-1 $\beta$, and TNF- $\alpha$ in BV-2 cells (20-22). Although we previously demonstrated that Plexin-A1 is involved in LPS-induced NO production in primary microglia (unpublished data), the involvement of Plexin-A1 in the expression of other TLR4-induced inflammatory mediators has yet to be adequately examined. In the present study, we examined the role of Plexin-A1 in inflammatory factor production in response to TLR4 activation in BV-2 cells. After BV-2 cells were treated with Plexin-A1-specific siRNA and cultivated for 1 or 2 days, the knockdown efficiency of Plexin-A1 was confirmed using quantitative RT-PCR, and a significant decrease of Plexin-A1 mRNA was observed in the BV-2 cells that were cultured for 2 days after siRNA treatment (Fig. 2A). To examine the effect of Plexin-A1 on TLR4 expression, TLR4 expression level was assessed using western blotting in Plexin-A1 knockdown cells; however, no significant change was observed (Fig. 2B). To examine the role of Plexin-A1 in the LPS-TLR4 signaling pathway in BV-2 cells, the expression levels of iNOS, IL-1 $\beta$ and TNF- $\alpha$ were assessed with quantitative RT-PCR in Plexin-A1 knockdown cells treated with vehicle or LPS. During TLR4 

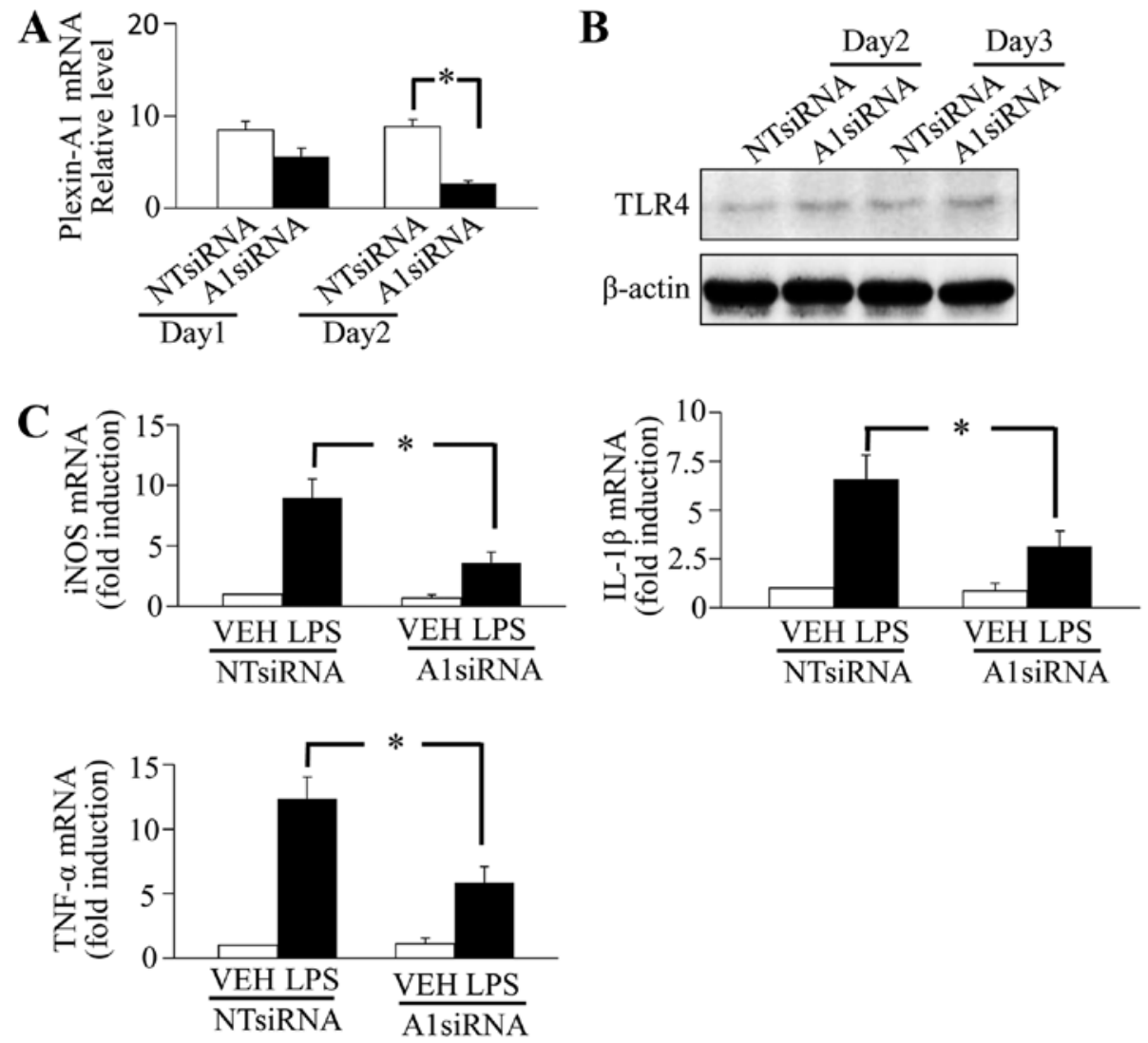

Figure 2. Plexin-A1 is required for the increased expression of inducible nitric oxide synthase (iNOS), interleukin-1 (IL-1 $\beta$ ) and tumor necrosis factor- $\alpha$ (TNF- $\alpha$ ) in lipopolysaccharide (LPS)-stimulated microglia. (A) Quantitative RT-PCR confirms the knockdown of Plexin-A1 expression by the transduction of the PlexinA1-specific siRNA in BV-2 cells. NT, non-target. Each column is the means \pm SEM. ${ }^{*} \mathrm{P}<0.05$, ANOVA. (B) The expression of Toll-like receptor 4 (TLR4) protein is not significantly decreased in Plexin-A1-specific siRNA-treated BV-2 cells compared with control siRNA-treated cells. (C) Addition of LPS to BV-2 microglia significantly increases the mRNA expression of iNOS, IL-1 $\beta$ and TNF- $\alpha$. Knockdown of Plexin-A1 expression with Plexin-A1-specific siRNA significantly inhibits LPS-induced elevations in iNOS, IL-1 $\beta$ and TNF- $\alpha$ mRNA in BV-2 cells. VEH, vehicle; LPS, lipopolysaccharide; NT siRNA, control non-target siRNA; A1 siRNA, Plexin-A1-specific siRNA.

stimulation with LPS, a significantly lower amount of iNOS, IL- $1 \beta$ and TNF- $\alpha$ mRNA was generated in BV-2 cells with Plexin-A1 knockdown than BV-2 cells treated with control non-target (NT) siRNA (Fig. 2C). These findings showed that Plexin-A1 is required for the TLR4-mediated generation of proinflammatory factors in BV-2 cells. Assessment of LPS-induced iNOS protein expression using western blotting showed a significantly lower level of iNOS protein expression in BV-2 cells with targeted silencing of Plexin-A1 than in control siRNA-treated BV-2 cells (Fig. 3A and B). Assessment of LPS-induced NO production by the Griess reaction revealed a significant decrease in NO in Plexin-A1-specific siRNAtreated BV-2 cells compared with control siRNA-treated cells (Fig. 3C). MTT assay showed no significant differences in cell viability among any BV-2 cell treatments (Fig. 3D).

Plexin-Al mediates the activation of $N F-\kappa B$ and ERK. During the microglial response to TLR agonists and microbial pathogens, NF- $\kappa \mathrm{B}$ and mitogen-activated protein kinase (MAPK) signaling pathways are known to regulate the production of inflammatory mediators (23-26). Since the generation of iNOS, IL-1 $\beta$, and TNF- $\alpha$ was decreased by Plexin-A1 knockdown (Figs. 2C and 3), we investigated the role of Plexin-A1 in these signaling pathways in BV-2 microglial cells. In a previous study, we reported that Sema3A-Plexin-A1 signaling increased NO production through crosstalk with the TLR4 pathway in primary microglia (18). Sema4D-Plexin-B1 signaling also increased NO production through ERK1/2 activation, which is dependent on inflammatory stimulation in primary microglia (17). Activation of ERK1/2 also plays a crucial role in the induction of iNOS expression in TLR4-stimulated microglia (27). In neuronal cells, Plexin-A1 is involved in the determination of the direction of axonal extension and the induction of growth cone collapse in an ERK1/2 activation-dependent manner through crosstalk with Plexin-B1 (28-32). Plexin-A1-mediated signaling in microglia may therefore be involved in the production of inflammatory factors through ERK1/2 activation by cooperating with the TLR4 pathway. Accordingly, to examine whether Plexin-A1 is involved in the activation of NF- $\kappa$ B and ERK1/2 in LPS-stimulated microglia, the phosphorylation state of these proteins was assessed in vehicle- or LPS-treated BV-2 cells with either control siRNA or Plexin-A1-specific siRNA. Control NT siRNA-treated BV-2 cells exhibited enhanced phosphorylation of NF- $\kappa$ B, I $\kappa$ B- $\alpha$, and ERK1/2 after LPS stimulation. In contrast, BV-2 cells with targeted knockdown of Plexin-A1 exhibited a significant decrease in NF- $\kappa \mathrm{B}$ and I $\kappa \mathrm{B}-\alpha$ phosphorylation compared with cells treated with control siRNA (Fig. 4). LPS stimulation induced a significant increase of ERK1/2 phosphorylation in control siRNA-treated BV-2 cells, but not in Plexin-A1-specific siRNA-treated BV-2 cells (Fig. 4). These 

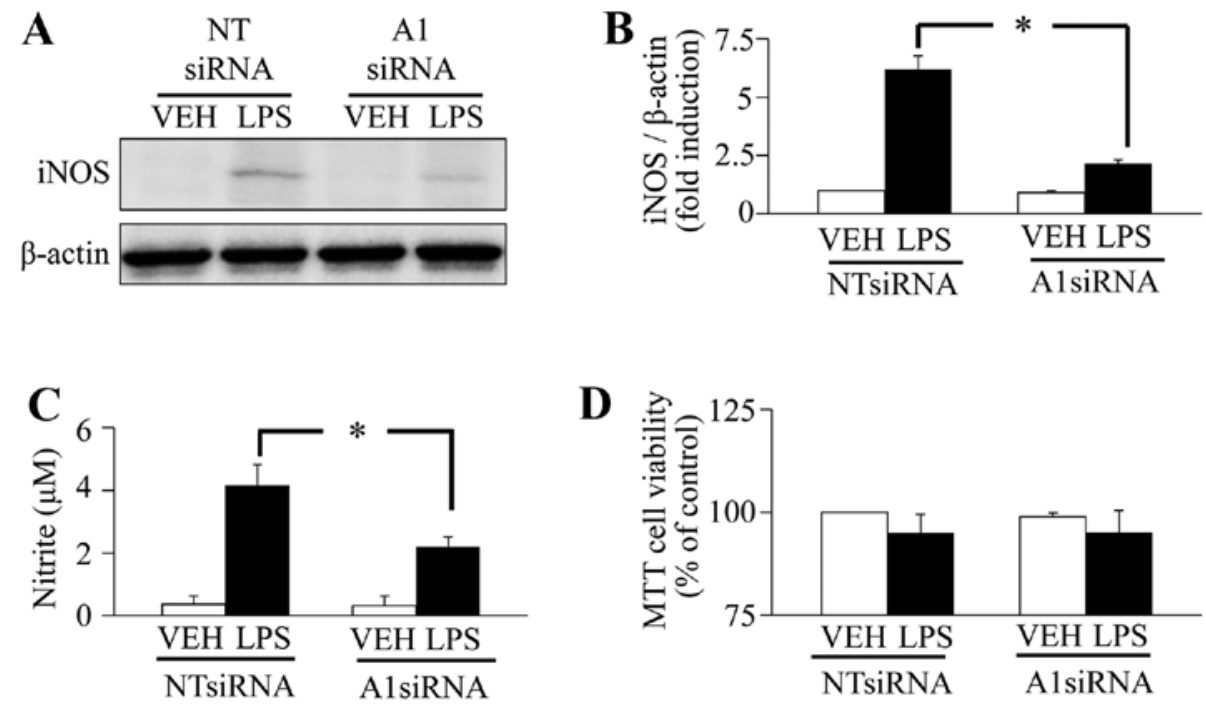

Figure 3. Plexin-A1 is required for NO production in LPS-stimulated microglia. (A) Expression level of iNOS protein in response to LPS compared between Plexin-A1 siRNA-treated and NT siRNA-treated BV-2 cells by western blotting. (B) Quantitative western blot analysis shows a significantly decreased expression of iNOS protein in LPS- and Plexin-A1 siRNA-treated BV-2 cells compared with LPS- and NT siRNA-treated BV-2 cells. (C) Nitrite concentrations in the culture supernatants determined by the Griess reaction. NO level in culture supernatant is significantly decreased in LPS- and Plexin-A1 siRNA-treated BV-2 cells compared with LPS- and NT siRNA-treated BV-2 cells. (D) Viability of BV-2 cells evaluated by MTT assay. Cell viabilities are not significantly different among any treatment group. Data are shown as mean \pm SEM. "P<0.05, ANOVA. To quantify iNOS or NO production, the cells were stimulated with or without LPS $(100 \mathrm{ng} / \mathrm{ml})$ for $6 \mathrm{~h}$ for quantitative RT-PCR or for $18 \mathrm{~h}$ for the Griess reaction. Data are representative of at least three independent experiments. VEH, vehicle; LPS, lipopolysaccharide; NT siRNA, control non-target siRNA; A1 siRNA, Plexin-A1-specific siRNA.

$\mathbf{A}$

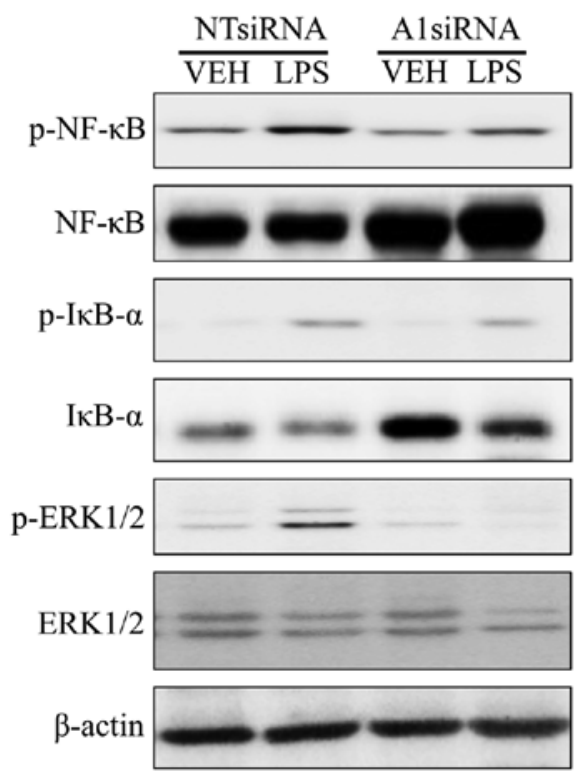

B
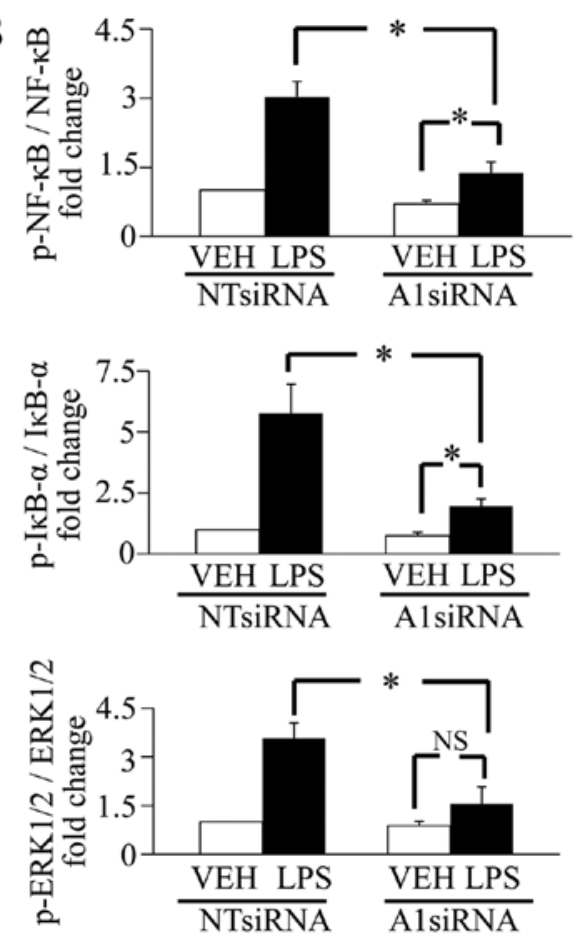

Figure 4. Plexin-A1 is required for LPS-induced activation of nuclear factor- $\kappa \mathrm{B}(\mathrm{NF}-\mathrm{\kappa} B)$ and extracellular signal-regulated kinase (ERK)1/2. (A) The figure shows western blot analysis of NF- $\mathrm{KB}$ and mitogen-activated protein kinase (MAPK) signaling molecules in BV-2 cells having control or Plexin-A1 siRNA nonstimulated or stimulated for $60 \mathrm{~min}$ with $100 \mathrm{ng} / \mathrm{ml}$ LPS. To quantify the immunoblots densitometry analysis was conducted on the basis of three independent

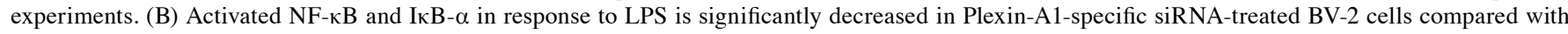
NT siRNA-treated BV-2 cells. Activated ERK1/2 in response to LPS stimulation is not significantly increased in Plexin-A1-specific siRNA-treated BV-2 cells compared with NT siRNA-treated BV-2 cells. Results are expressed as mean \pm SEM. "P<0.05. VEH, vehicle; LPS, lipopolysaccharide; NT siRNA, control non-target siRNA; A1 siRNA, Plexin-A1-specific siRNA.

findings indicate that Plexin-A1 deficiency leads to a significant defect in NF- $\mathrm{KB}$ and ERK1/2 activation in TLR4-mediated signaling in BV-2 microglial cells.
Sema3A enhances LPS-induced nitrite production through ERK activation in a Plexin-A1-dependent manner. Since Plexin-A1 knockdown suppressed the expression of 

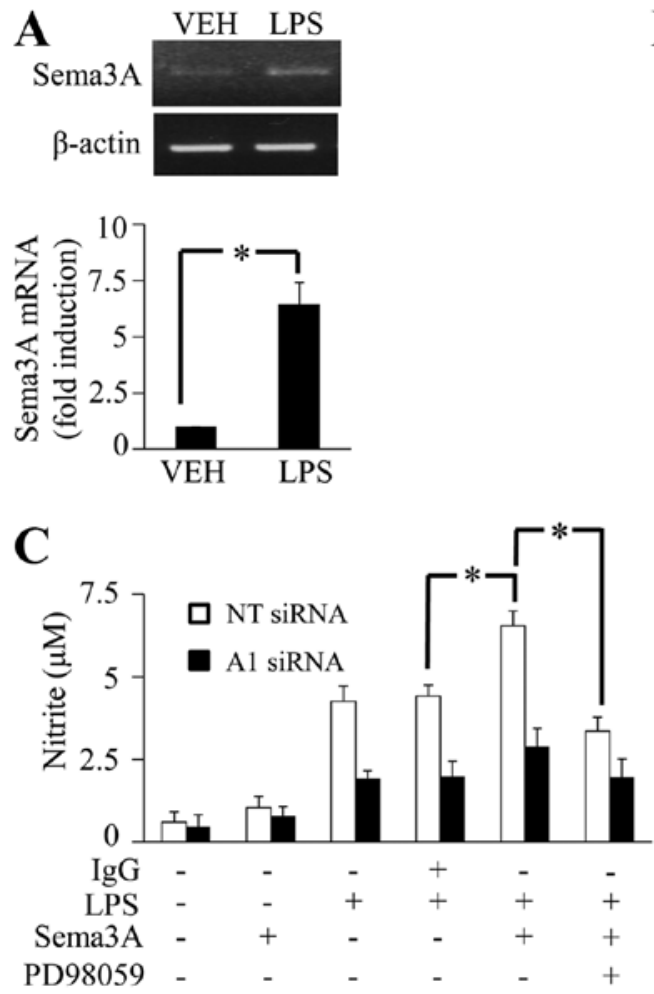

B

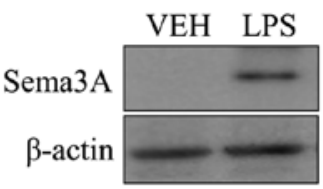

Supernatant

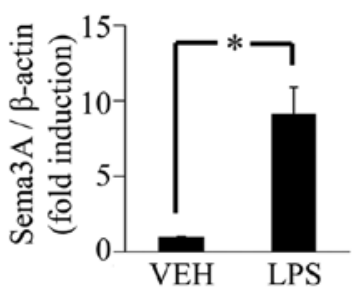

D

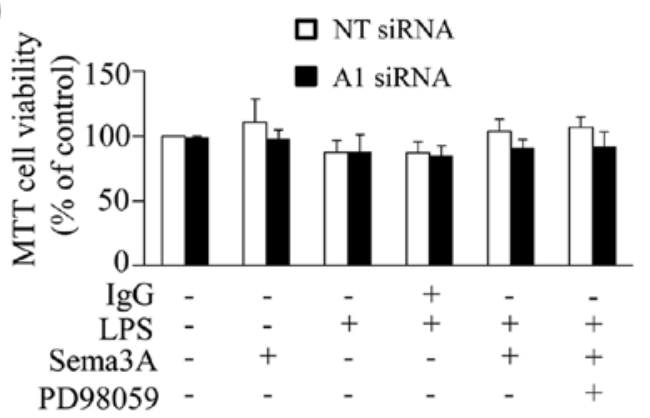

Figure 5. Sema3A enhances LPS-induced nitrite production through ERK activation in a Plexin-A1-dependent manner. (A and B) BV-2 cells were left untreated or were treated with $100 \mathrm{ng} / \mathrm{ml}$ LPS for (A) $2 \mathrm{~h}$ or (B) $4 \mathrm{~h}$. (A) Sema3A mRNA and (B) Sema3A protein were examined by RT-PCR or western blot analysis. The expression of Sema3A mRNA and protein is significantly increased in LPS-treated BV-2 microglia. *P<0.05 compared with untreated BV-2 cells. (C) BV-2 cells transduced with control or Plexin-A1-specific siRNA were treated with or without PD98059 in the presence of LPS (100 ng/ml) plus Sema3A (1 $\mu \mathrm{g} / \mathrm{ml})$ for $18 \mathrm{~h}$. Nitrite production was quantified by the Griess reaction. NO level in medium supernatant of Sema3A-treated BV-2 cells stimulated with LPS is significantly increased compared with IgG-treated BV-2 stimulated with LPS. However, the significant increase in NO was suppressed by pre-treatment with ERK inhibitor PD98059. IgG fragment $(1 \mu \mathrm{g} / \mathrm{ml})$ was added as a negative control. $\mathrm{P}<0.05$ compared with control. (D) Cell viability shows no significant change among any group as measured by MTT assay. VEH, vehicle; LPS, lipopolysaccharide; NT siRNA, control non-target siRNA; A1 siRNA, Plexin-A1-specific siRNA.

LPS-induced inflammatory mediators, the BV-2 cell itself may produce the Plexin-A1 ligand Sema3A. A possible mechanism may be that the production and/or secretion of Sema3A in BV-2 cells is facilitated by LPS, after which Sema3A binds with Plexin-A1 to activate BV-2 cells in an autocrine manner. To test the possibility, BV-2 cells were treated with LPS and examined for evidence of Sema3A mRNA production and Sema3A protein secretion in the culture medium. Two-hour LPS treatment of BV-2 cells significantly increased Sema3A mRNA levels (Fig. 5A). Furthermore, 4-h LPS treatment of $\mathrm{BV}-2$ cells induced the appearance of proteolytically processed Sema3A (65 kDa; Fig. 5B) in the supernatant, as shown in other experimental systems (33-35). The data suggest that the production and secretion of Sema3A induced by the TLR4 activation of BV-2 cells may cause Plexin-A1 to function in a signaling loop, thereby enhancing inflammatory signals and cytokine production. The above results suggested that Sema3A-Plexin-A1 signaling is involved in the increase of LPS-induced NO production through ERK1/2 in BV-2 cells (Figs. 3 and 4). Thus, BV-2 cells transduced with control NT or Plexin-A1 siRNA were treated with Sema3A in the presence $(100 \mathrm{ng} / \mathrm{ml})$ or absence of LPS to functionally examine whether Sema3A functions as a ligand for Plexin-A1 in BV-2 cells. The Sema3A ligand was composed of Sema3A fused with the murine $\operatorname{IgG} 2 \mathrm{~A}$ Fc fragment, and the $\operatorname{IgG} 2 \mathrm{~A}$ Fc fragment alone was utilized as a negative control. In the presence of LPS, nitrite levels were increased (Fig. 5C); in its absence, Sema3A did not induce nitrite production in BV-2 cells. Following the addition of soluble Sema3A (Fig. 5C), LPS-induced NO production was intensified in BV-2 cells transduced with NT siRNA. By contrast, the addition of the IgG2A Fc control made no effect. Enhancement of LPS responsiveness was not observed in BV-2 cells with Plexin-A1 knockdown (Fig. 5C). Accordingly it is indicated that Sema3A ligation to Plexin-A1 intensifies NO generation of BV-2 cells only in the presence of LPS, which suggests that in BV-2 cells, both LPS and Sema3A are necessary for the establishment of optimal intracellular signaling to produce inflammatory mediators. To clarify whether the increase in NO production from Sema3A-Plexin-A1 signaling is dependent on the activation of ERK1/2, we examined whether treatment with ERK1/2 inhibitor suppressed the increase in LPS-induced NO production following the addition of Sema3A in BV-2 cells. BV-2 cells treated with LPS, Sema3A, and ERK1/2 inhibitor showed significantly decreased NO production, as compared with BV-2 cells treated with only LPS and Sema3A (Fig. 5C). These findings indicated that Sema3A-Plexin-A1 signaling was involved in the increased production of NO through ERK1/2 activation in the microglial response to LPS. These in vitro results suggested that Plexin-A1 binding by Sema3A is synergized with LPS engagement of TLR4 on microglia to expand innate inflammatory responses in the brain. 


\section{Discussion}

Findingsofthisstudy haveshownthreenovelfindingsconcerning the roles of Plexin-A1 expressed in BV2 microglial cells. First, Plexin-A1 enhances the LPS-induced increase in inflammatory mediators in BV-2 cells, suggesting that Plexin-A1 plays a crucial role in the activation process of microglia. Second, the results clarified that Plexin-A1 in BV-2 cells is involved in the activation of $N F-\kappa B$ and MAPK pathway in the LPS response. Third, the results indicate that Sema3A-Plexin-A1 signaling increases LPS-induced NO production through ERK activation in BV-2 cells, thereby demonstrating that part of the intracellular Sema3A-Plexin-A1 signaling pathway operates in the activation of microglia. Results of a previous study (18) have shown that Sema3A-Plexin-A1 signaling increases NO production through crosstalk with TLR4 signaling in primary microglia. However, the mechanisms involved in the intracellular signaling pathway were not identified. Therefore, to the best of our knowledge, this is the first study showing the crucial involvement of Sema3A-Plexin-A1 signaling in the LPS-induced production of inflammatory mediators through $\mathrm{NF}-\kappa \mathrm{B}$ and ERK activation in microglia.

We confirmed the expression of Plexin-A1 and Neuropilin-1 with RT-PCR and western blotting in BV-2 cells (Fig. 1). The binding of Sema3A to the receptor complex of Neuropilin-1 and Plexin-A1 on the cell membrane transduces intracellular signaling (36). Our earlier study has confirmed the involvement of Sema3A-Plexin-A1 signaling in increased LPS-induced NO production in primary microglia (18). Similar to primary microglia, LPS-stimulated BV-2 cells increase the expression of inflammatory mediators such as iNOS, IL-1 $\beta$ and TNF- $\alpha$ (20-22), although previous studies have not directly demonstrated that Plexin-A1 is involved in the induction of inflammatory mediators by the stimulation of TLR4. To investigate the role of Plexin-A1 in BV-2 cells stimulated by LPS, Plexin-A1 was knocked down using Plexin-A1-specific siRNA and the expression level of inflammatory mediators was assessed in microglia with TLR4 activation. As a result, Plexin-A1-specific siRNA-treated BV-2 cells showed a significant decrease in iNOS, IL- $1 \beta$ and TNF- $\alpha$ mRNA expression in LPS-treated cells as compared with control siRNA-treated cells (Fig. 2C). Quantification of the iNOS protein with western blotting and NO in the culture supernatant using the Griess reaction showed significant decreases of iNOS and NO levels in Plexin-A1-specific siRNA treated-BV-2 cells compared with control siRNA-treated cells (Fig. 3A-C). Since the assessment of cell viability by MTT assay did not show any significant differences between Plexin-A1-specific siRNA-treated and control NT siRNA-treated BV-2 cells, the significant decrease in NO production may not reflect group-based differences of cell viability. Accordingly Plexin-A1 is required to increase the expression of inflammatory mediators in LPS-stimulated BV-2 cells.

During the microglial response to microbial pathogens and TLR agonists, MAPK and NF- $\kappa$ B signaling pathways are known to regulate the production of inflammatory mediators (23-26). The decreased generation of iNOS, IL- $1 \beta$ and TNF- $\alpha$ after targeted silencing of Plexin-A1 led to the investigation of the potential role of Plexin-A1 in these signaling pathways (Figs. 2C, 3A and B). Previously, we clarified that Sema3A-
Plexin-A1 signaling is involved in NO production in primary microglia by crosstalk with TLR4-mediated signaling (18). Activation of ERK1/2 plays a crucial role in the induction of iNOS expression in TLR4-stimulated microglia (27). Sema4DPlexin-B1 signaling increases NO production through ERK1/2 activation dependent on inflammatory stimulation in primary microglia (17). In neuronal cells, Plexin-A1 is involved in determining the direction of axonal extension and growth cone collapse in an ERK1/2 activation-dependent manner through crosstalk with Plexin-B1 (28-32). In microglial cells, therefore, Plexin-A1 signaling may be involved in the production of inflammatory factors through ERK1/2 activation and cooperative action with the TLR4 pathway. To clarify the intracellular signaling molecules located downstream of Plexin-A1 signaling that have crosstalk with the TLR4 pathway in microglial activation, we assessed the activation level of NF- $\kappa \mathrm{B}$, I $\kappa \mathrm{B}-\alpha$ and ERK1/2 by western blotting. Phosphorylated NF- $\kappa B$ and I $\kappa \mathrm{B}-\alpha$ significantly increased in control siRNA-treated BV-2 cells $1 \mathrm{~h}$ after LPS stimulation as compared with Plexin-A1specific siRNA-treated BV2 cells. These findings suggest that Plexin-A1 is involved in the phosphorylation of NF- $\kappa$ B and I $\mathrm{B}-\alpha$ in the LPS response of BV-2 cells. In Plexin-A1-specific siRNA-treated BV-2 cells, LPS treatment induced a significant increase of phosphorylated NF- $\kappa \mathrm{B}$ and $\mathrm{I} \kappa \mathrm{B}-\alpha$ compared with vehicle-treated cells (Fig. 4). This result may be explained by the imperfect removal of Plexin-A1 or the significant phosphorylation of $\mathrm{NF}-\kappa \mathrm{B}$ and $\mathrm{I} \kappa \mathrm{B}-\alpha$ through a pathway other than that of Plexin-A1 signaling. Sema3A secreted from macrophages enhances the LPS response by acting in an autocrine manner to activate Plexin-A4-mediated signaling (16). Therefore, the involvement of Plexin-A4 signaling in LPS-dependent activation in microglia may be a useful explanation for the fact that even in cells with targeted silencing of Plexin-A1, phosphorylated NF- $\kappa \mathrm{B}$ and $\mathrm{I} \kappa \mathrm{B}-\alpha$ were significantly increased with LPS treatment compared with vehicle treatment. Since Plexin-A1 signaling may strengthen microglial activation through ERK activation in response to LPS, the level of phosphorylation of ERK was quantified by western blotting. Phosphorylated ERK was found to increase significantly in control siRNA-treated BV-2 cells compared with Plexin-A1-specific siRNA-treated cells. This result suggests that Plexin-A1 may be involved in LPS-induced microglial activation through ERK phosphorylation. Since the phosphorylation of ERK $1 / 2$ in LPS treatment of Plexin-A1-specific siRNA-treated BV-2 cells was not found to be significantly greater than in the vehicle-treated BV-2 cells with Plexin-A1 knockdown, phosphorylation of ERK in the LPS response may be dependent on Plexin-A1. In the LPS response of macrophages, Plexin-A4-mediated signaling is involved in c-jun N-terminal kinase phosphorylation (16), suggesting that plexin receptors may increase the LPS response through differential pathways, depending on various cell types or receptor families. Thus, Plexin-A1 is crucial for the amplification of the LPS response in microglia.

In BV-2 microglial cells, Sema3A was found to be secreted by the cell itself in an autocrine manner, as in macrophages (Fig. 4B). Whereas injured neurons are suggested to produce Sema3A and Sema3A induces microglial apoptosis (37), microglia are also suggested to produce Sema3A which appears to increase LPS-induced microglial activation through Plexin-A1-mediated signaling. In control siRNA- 
treated BV-2 cells, Sema3A generated a significant increase of LPS-induced NO production as compared with control IgG, and this increase was significantly suppressed by pretreatment with ERK inhibitor. By contrast, NO levels did not increase significantly in BV-2 cells with targeted knockdown of Plexin-A1 even after treatment with Sema3A, when compared with the IgG treatment. These results suggest that Sema3APlexin-A1 signaling is involved in the increase of LPS-induced NO production in activated microglia through ERK activation. Therefore, both Plexin-A1 and Sema3A are potential targets for the treatment of encephalopathy triggered by LPS and neuroinflammation-related mental disorders.

\section{Acknowledgements}

We would like to thank the members of the Department of Physiology of Meijo University for their helpful discussion and technical assistance. The study was primarily supported by a Grant-in-Aid for Scientific Research from the Ministry of Education, Science, Sports and Culture, Japan (No. 22590195), and research grants from Meijo University.

\section{References}

1. Ransohoff RM and Perry VH: Microglial physiology: unique stimuli, specialized responses. Annu Rev Immunol 27: 119-145, 2009 .

2. Heppner FL, Greter M, Marino D, et al: Experimental autoimmune encephalomyelitis repressed by microglial paralysis. Nat Med 11: 146-152, 2005

3. Jack C, Ruffini F, Bar-Or A and Antel JP: Microglia and multiple sclerosis. J Neurosci Res 81: 363-373, 2005.

4. Ponomarev ED, Shriver LP and Dittel BN: CD40 expression by microglial cells is required for their completion of a two-step activation process during central nervous system autoimmune inflammation. J Immunol 176: 1402-1410, 2006.

5. Lee SJ and Lee S: Toll-like receptors and inflammation in the CNS Curr Drug Targets Inflamm Allergy 1: 181-191, 2002.

6. Tran TS, Kolodkin AL and Bharadwaj R: Semaphorin regulation of cellular morphology. Annu Rev Cell Dev Biol 23: 263-292, 2007.

7. Gu Y, Filippi MD, Cancelas JA, et al: Hematopoietic cell regulation by Rac1 and Rac2 guanosine triphosphatases. Science 302 445-449, 2003.

8. Toyofuku T,Zhang H, Kumanogoh A, et al: Guidance of myocardial patterning in cardiac development by Sema6D reverse signalling. Nat Cell Biol 6: 1204-1211, 2004.

9. Neufeld G and Kessler O: The semaphorins: versatile regulators of tumour progression and tumour angiogenesis. Nat Rev Cancer 8: 632-645, 2008.

10. Suzuki K, Kumanogoh A and Kikutani H: Semaphorins and their receptors in immune cell interactions. Nat Immunol 9: 17-23, 2008.

11. Walzer T, Galibert L and De Smedt T: Dendritic cell function in mice lacking Plexin C1. Int Immunol 17: 943-950, 2005.

12. Choi YI, Duke-Cohan JS, Ahmed WB, et al: PlexinD1 glycoprotein controls migration of positively selected thymocytes into the medulla. Immunity 29: 888-898, 2008.

13. Yamamoto M, Suzuki K, Okuno T, et al: Plexin-A4 negatively regulates T lymphocyte responses. Int Immunol 20: 413-420, 2008

14. Wong AW, Brickey WJ, Taxman DJ, et al: CIITA-regulated plexin-A1 affects T-cell-dendritic cell interactions. Nat Immunol 4: 891-898, 2003.

15. Takegahara N, Takamatsu H, Toyofuku T, et al: Plexin-A1 and its interaction with DAP12 in immune responses and bone homeostasis. Nat Cell Biol 8: 615-622, 2006.

16. Wen H, Lei Y, Eun SY and Ting JP: Plexin-A4-semaphorin 3A signaling is required for Toll-like receptor- and sepsis-induced cytokine storm. J Exp Med 207: 2943-2957, 2010.
17. Okuno T,Nakatsuji Y,Moriya M, et al: Roles of Sema4D-plexin-B1 interactions in the central nervous system for pathogenesis of experimental autoimmune encephalomyelitis. J Immunol 184: 1499-1506, 2010

18. Ito T, Yoshida K, Negishi T, et al: Plexin-A1is required for Toll-like receptor-mediated microglial activation in the development of lipopolysaccharide-induced encephalopathy. Int J Mol Med 33: 1122-1130, 2014

19. Blasi E, Barluzzi R, Bocchini V, Mazzolla R and Bistoni F: Immortalization of murine microglial cells by a v-raf/v-myc carrying retrovirus. J Neuroimmunol 27: 229-237, 1990.

20. Zeng KW, Wang S, Dong X, Jiang Y and Tu PF: Sesquiterpene dimer (DSF-52) from Artemisia argyi inhibits microglia-mediated neuroinflammation via suppression of NF- $\mathrm{BB}, \mathrm{JNK} / \mathrm{p} 38$ MAPKs and Jak2/Stat3 signaling pathways. Phytomedicine 21: 298-306, 2013.

21. Manivannan J, Tay SS, Ling EA and Dheen ST: Dihydropyrimidinase-like 3 regulates the inflammatory response of activated microglia. Neuroscience 253: 40-54, 2013

22. Oh WJ, Jung U, Eom HS, Shin HJ and Park HR: Inhibition of lipopolysaccharide-induced proinflammatory responses by Buddleja officinalis extract in BV-2 microglial cells via negative regulation of NF- $\mathrm{KB}$ and ERK1/2 signaling. Molecules 18: 9195-9206, 2013.

23. Kaminska B: MAPK signalling pathways as molecular targets for anti-inflammatory therapy - from molecular mechanisms to therapeutic benefits. Biochim Biophys Acta 1754: 253-262, 2005.

24. Kaminska B, Gozdz A, Zawadzka M, Ellert-Miklaszewska A and Lipko M: MAPK signal transduction underlying brain inflammation and gliosis as therapeutic target. Anat Rec (Hoboken) 292: 1902-1913, 2009.

25. Koistinaho $M$ and Koistinaho J: Role of p38 and p44/42 mitogenactivated protein kinases in microglia. Glia 40: 175-183, 2002.

26. Johnson GL and Lapadat R: Mitogen-activated protein kinase pathways mediated by ERK, JNK, and p38 protein kinases. Science 298: 1911-1912, 2002.

27. Bhat NR, Zhang P, Lee JC and Hogan EL: Extracellular signal regulated kinase and $\mathrm{p} 38$ subgroups of mitogen-activated protein kinases regulate inducible nitric oxide synthase and tumor necrosis factor- $\alpha$ gene expression in endotoxin-stimulated primary glial cultures. J Neurosci 18: 1633-1641, 1998.

28. Bechara A, Nawabi H, Moret F, et al: FAK-MAPK-dependent adhesion disassembly downstream of L1 contributes to semaphorin3A-induced collapse. EMBO J 27: 1549-1562, 2008.

29. Lerman O, Ben-Zvi A, Yagil Z and Behar O: Semaphorin3A accelerates neuronal polarity in vitro and in its absence the orientation of DRG neuronal polarity in vivo is distorted. Mol Cell Neurosci 36: 222-234, 2007.

30. Bagnard D, Sainturet N, Meyronet D, et al: Differential MAP kinases activation during semaphorin3A-induced repulsion or apoptosis of neural progenitor cells. Mol Cell Neurosci 25: 722-731, 2004.

31. Campbell DS and Holt CE: Apoptotic pathway and MAPKs differentially regulate chemotropic responses of retinal growth cones. Neuron 37: 939-952, 2003

32. Kruger RP, Aurandt J and Guan KL: Semaphorins command cells to move. Nat Rev Mol Cell Biol 6: 789-800, 2005.

33. Adams RH, Lohrum M, Klostermann A, Betz H and Püschel AW: The chemorepulsive activity of secreted semaphorins is regulated by furin-dependent proteolytic processing. EMBO J 16: 6077-6086, 1997.

34. Catalano A, Caprari P, Moretti S, Faronato M, Tamagnone L and Procopio A: Semaphorin-3A is expressed by tumor cells and alters T-cell signal transduction and function. Blood 107: 3321-3329, 2006.

35. LepelletierY,Moura IC,Hadj-Slimane R, et al: Immunosuppressive role of semaphorin-3A on T cell proliferation is mediated by inhibition of actin cytoskeleton reorganization. Eur J Immunol 36: 1782-1793, 2006

36. Banks WA and Erickson MA: The blood-brain barrier and immune function and dysfunction. Neurobiol Dis 37: 26-32, 2010.

37. Majed HH, Chandran S, Niclou SP, et al: A novel role for Sema3A in neuroprotection from injury mediated by activated microglia. J Neurosci 26: 1730-1738, 2006. 